\title{
Novel Synthesis of Amphiphilic Core-Shell Type Gel
}

\author{
Takashi IrzawA ${ }^{\dagger}$ and Fumihiko Matsuda \\ Department of Chemical Engineering, Faculty of Engineering, Hiroshima University, \\ Kagamiyama, Higashi-Hiroshima 739, Japan
}

(Received June 14, 1997)

\begin{abstract}
KEY WORDS Gel Capsule / Esterification / 1,8-Diazabicyclo-[5,4,0]-7-undecene / Poly(acrylic acid) / Chemical Release /
\end{abstract}

Poly(acrylic acid) gel is a good for super waterabsorbing gel. ${ }^{1}$ This gel can store many water-soluble chemicals. However, it is not used widely for storage and release of chemicals because the gel swells in water and is easily broken. To remove this defect, we propose new amphiphilic core-shell type gel "gel capsule" consisting of a hydrophilic core gel covered and bound chemically with hydrophobic shell layer. This hydrophobic layer does not only reinforce the gel, but it controls the chemical release and occlusion. In a previous paper, ${ }^{2}$ a new gel capsule was successfully synthesized by the selective thermal acid-catalyzed deesterification of internal poly( $t$-butyl methacrylate) gel in a solvent. The gel capsule can be prepared by esterification of poly(carboxylic acid) gels. However, the esterification is very difficult, because the polymer is hydrophilic and the resulting ester is hydrophobic, and both polymers are soluble in few common solvents. Phase transfer catalyst (PTC) is a powerful method for heterogeneous esterification of low molecular weight carboxylic acid with alkyl halide and polymer containing halogen. ${ }^{3}$ However, PTC does not work well for the esterification of poly(carboxylic acid) with alkyl halide. ${ }^{3 b}$ Shimokawa ${ }^{4}$ and Iizawa et $a l .{ }^{5}$ have studied the reactions of pendant carboxylic acids in polymers with alkyl halides using 1,8-diazabicyclo-[5,4,0]-7-undecene (DBU) as an acid acceptor. The quantitative esterification was achieved by this method in an aprotic polar solvent under mild conditions.

This communication reports the esterification of hydrophilic DBU salt (DAA) of poly(acrylic acid) gel with alkyl halide in a solvent. Esterification from the outer gives a new core-shell type gel consisting of a hydrophilic unreacted core and hydrophobic esterified shell (Scheme 1). The esterification causes polarity and affinity for chemicals to change dramatically.

\section{EXPERIMENTAL}

\section{Synthesis of DAA}

Acrylic acid $(75 \mathrm{~g})$ and $N, N^{\prime}$-methylenebisacrylamide $(0.53 \mathrm{~g})$ were dissolved in water $(90 \mathrm{~mL})$. The solution was charged in a separable flask with dozens of Teflon tubes (internal diameter: $8 \mathrm{~mm}$, length: about $6 \mathrm{~cm}$ ). To the solution were added potassium peroxodisulfate $(0.188 \mathrm{~g})$ in water $(30 \mathrm{~mL})$ and sodium sulfite $(0.175 \mathrm{~g})$ in water $(30 \mathrm{~mL})$ under nitrogen current. The reaction mixture was stirred for $10 \mathrm{~min}$, and stood for overnight at room temperature and during this period nearly $100 \%$ of the monomer was polymerized to form a polymer gel.
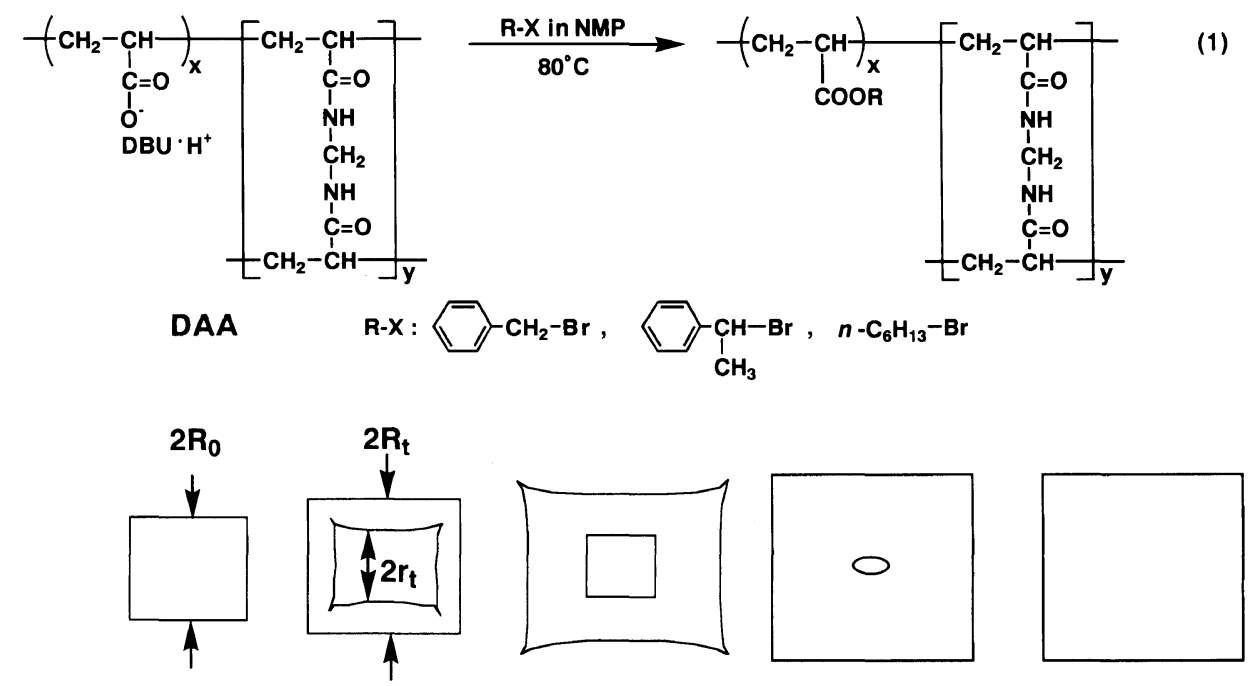

$r_{t} / R_{0}=1.0$

$1.0-0.7$

$0.7-0.1$

0.1 - 0

0

Scheme 1. Reaction of DAA with alkyl halide.

\footnotetext{
† To whom all correspondence should be addressed.
} 
After completion of gelation, the gel bars in the tubes were taken out of the gel in the flask. The gel bars with tubes were cut to give cylindrical samples of gel $8 \mathrm{~mm}$ long. The sample was pushed out from the tube and immersed into a large amount of water and methanol to wash away unreacted substances. The sample was dipped into excess DBU solution in methanol at room temperature for 1 day, and washed with methanol. The gel was dried carefully and slowly, and finally dried in vacuo at $60^{\circ} \mathrm{C}$ until constant weight.

\section{Esterification of DAA and Its Measurement}

A typical esterification is as follows. Benzyl bromide $(5 \mathrm{~mL})$ and $N$-methyl-2-pyrrolidone (NMP, $40 \mathrm{~mL}$ ) were charged into a $50 \mathrm{~mL}$ cylindrical cell in a water bath at $80^{\circ} \mathrm{C}$. To the solution was added a DAA sample $(0.3008 \mathrm{~g}) . R_{t}$ and $r_{t}$ of the sample, the radii of shell and core after $t$ min, respectively, were periodically observed by a reading microscope. After the disappearance of core part, the gel was washed with acetone by a Soxhlet extractor and dried in vacuo at $110^{\circ} \mathrm{C}$ until constant weight. The weight of the esterified gel is $0.2092 \mathrm{~g}$. The degree of esterification is $92.0 \%$, was calculated from change of the weight before and after the reaction.

\section{Chemical Release from the Gel Capsule}

The gel capsule $\left(r_{t} / R_{0}=0.75\right)$ was dipped into a solution $\left(0.1 \mathrm{mmol} \mathrm{L}^{-1}\right)$ of Rose-Bengal in methanol-acetone mixture $(1: 1)$ and allowed to swell for 1 day at $30^{\circ} \mathrm{C}$. After drying, the shell layer was selectively washed with acetone by a Soxhlet extractor and dried. The sample was placed in $300 \mathrm{~mL}$ of aqueous $1 \mathrm{wt} \% \mathrm{NaCl}$ solution at $50^{\circ} \mathrm{C} . A_{t}$, absorbance of the solution at $548 \mathrm{~nm}$ after $t$ min, was measured on a UV photometer.

\section{RESULTS AND DISCUSSION}

DAA was prepared by the copolymerization of acrylic acid with $0.5 \mathrm{~mol} \%$ of $N, N^{\prime}$-methylenebisacrylamide in Teflon tubes and neutralization of the resulting gel with excess DBU in methanol. DAA did not swell in usual solvents except water, alcohols, and dimethylsulfoxide (DMSO). When the gel was placed in NMP containing excess benzyl bromide at $80^{\circ} \mathrm{C}$, swelling occurred and the gel was divided into a swollen shell and an unswollen core. The shell expanded with decreasing core size during reaction. At the end of the reaction, the core disappeared. The radius of shell increased gradually for a long time after the reaction was completed. The shell of the obtained gel did not swell in water and methanol, although the hydrophilic core swelled well in these solvents. The IR spectrum of the shell part is shown in Figure 1. The spectrum showed strong absorption at 1733 ( $\mathrm{C}=\mathrm{O}$, stretching), was different from that of poly(acrylic acid), and agreed with that of poly(benzyl acrylate). The degree of esterification of gels obtained from the reactions of DAA with benzyl bromide and hexyl bromide was 92.0 and $94.2 \mathrm{~mol} \%$, respectively, calculated from change of the weights before and after the reactions. Esterification is thus very high in the swollen parts, and the resulting gels are the corresponding poly(alkyl acrylate) gels with small amount of carboxylic acids. The reaction proceeded gradually from the surface

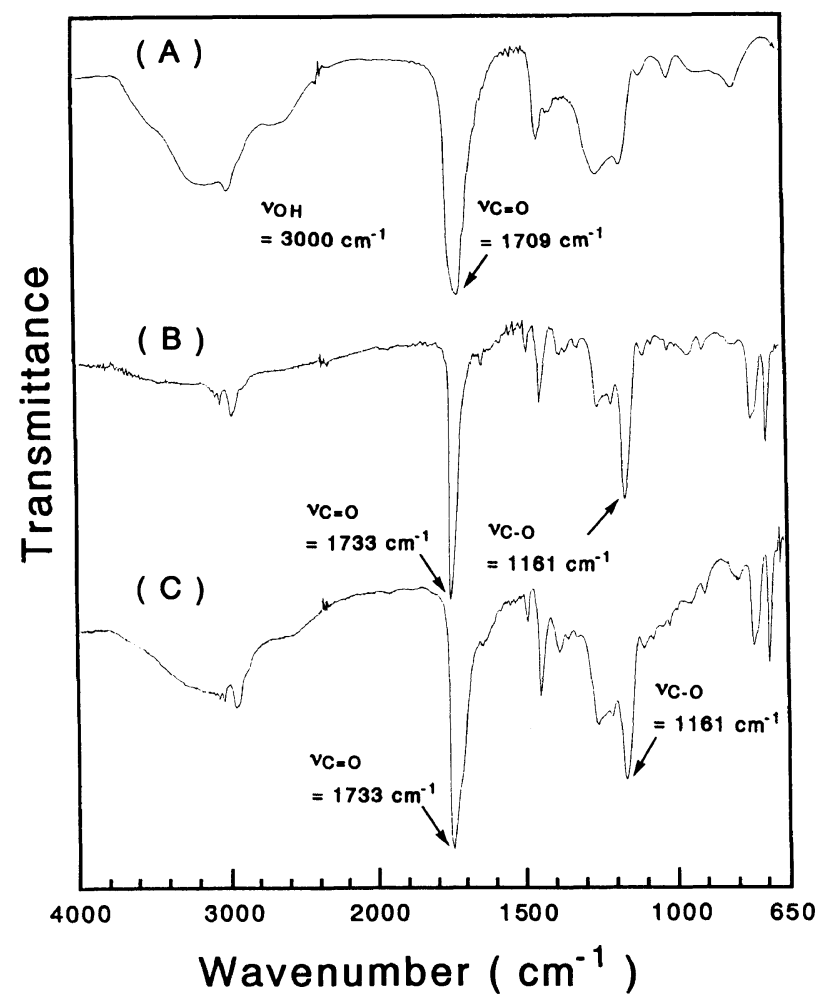

Figure 1. IR spectra of (A) soluble poly(acrylic acid), (B) soluble poly(benzyl acrylate), and (C) shell part of the core-shell type gel.

of the gel to the interior to give an amphiphilic core-shell. This reaction was observed at wide range of temperature in many solvents. DMSO is a good solvent for DAA and its esterified gel. When DAA swollen in DMSO was used, the reaction took place in a single gel phase. However, the gel was broken in the reaction because of the difference in swelling of the esterified and unreacted parts.

When a cylindrical DAA (length the same as diameter: about $7 \mathrm{~mm}$ ) was used, the cylindrical shape of core was similar to the original until high conversion. $R_{t}$ and $r_{t}$, radius of shell and core after $t$ min, respectively, were measured by a reading microscope. When the gel was considered infinite cylinder, the apparent ratio of reacted gel to the original gel $(x)$ was determined as function of $r_{t} / R_{0}$ (eq 2). The reaction can be followed as a function of $r_{t} / R_{0}$ (Figure 2). The thickness of the shell was easily controlled by the reaction time. Benzyl bromide is more reactive than hexyl bromide and 1-phenethyl bromide under homogeneous conditions. However, the reaction with benzyl bromide showed almost the same rate as that with hexyl bromide, although the reaction is faster than that of 1-phenethyl bromide. The observed reaction rate seamed to depend on the molecular size rather than the reactivity of the alkyl halides. This reaction is very slower compared with similar homogeneous reaction. ${ }^{5}$ These results suggest that the rate of the shrinking unreacted core is controlled by the diffusion of the alkyl halide or solvent in the shell layer. This reaction occurred at the outer skin of the unreacted core and proceeded by similar mechanism to unreacted core model for burning of cokes and roasting of ores. ${ }^{6}$ However, the overall reaction includes a complex mass transfer of solvent and alkyl halide. $R_{t}$ increases during the reaction and the shell layer is not uniformed. Therefore, the exact reaction 


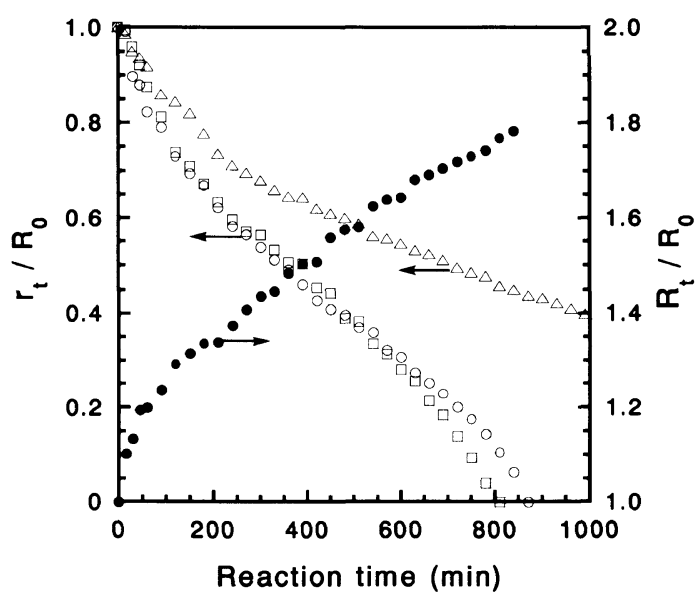

Figure 2. Reactions of DAA with various alkyl halides in NMP at $80^{\circ} \mathrm{C}$. Change of $\left(-R_{t}\right.$ during the reaction with benzyl bromide, and $r_{t}$ during reactions with $(\square)$ hexyl bromide, $(\bigcirc)$ benzyl bromide, and $(\triangle)$ 1-phenethyl bromide.

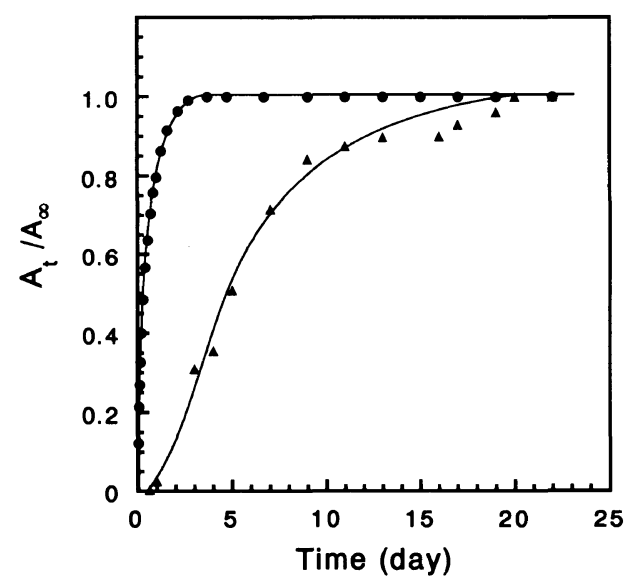

Figure 3. Change in absorbance of the aqueous solution with the gel: () DAA with Rose-Bengal; ( $\mathbf{\Delta})$ gel capsule $\left(r_{t} / R_{0}=0.75\right)$ containing Rose-Bengal in the core part.

mechanism cannot be explained from these results.

$$
x=1-\left(\frac{r_{t}}{R_{0}}\right)^{2}
$$

Release of Rose-Bengal from the gel capsule was carried out in aqueous $1 \mathrm{wt} \% \mathrm{NaCl}$ solution at $50^{\circ} \mathrm{C}$ as described previously. ${ }^{2}$ Typical release of Rose-Bengal is shown using unreacted DAA and the core-shell typed gel prepared from the reaction with hexyl bromide at $80^{\circ} \mathrm{C}$ for $1 \mathrm{~h}\left(r_{t} / R_{0}=0.75\right)$ in Figure 3 . When the gels containing Rose-Bengal were dipped in solution, the release of Rose-Bengal took place with swelling the gels. The swelling and release of core-shell typed gel were slower than those of DAA. This suggests that the shell layer may possibly work as a permeability barrier for many chemicals containing water.

From these results, it is concluded that successful synthesis of amphiphilic core-shell typed gel is by esterification of poly(acrylic acid) gel with alkyl halide using DBU. The reaction was markedly affected by the kinds of alkyl halide. The thickness of the shell and core layers was easily controlled by the reaction time. Further studies on the esterification of poly(acrylic acid) gels are now in progress to make the reaction mechanism and application clear.

Acknowledgment. This work was partly supported by a Grant-in-Aid for Scientific Research (No. 08651053) from the Ministry of Education, Science, and Culture of Japan, for which we are grateful.

\section{REFERENCES}

1. F. Masuda, "Kokyushu Kobunshi," Kyoritsu Shuppan Inc., Tokyo, 1987.

2. T. Iizawa, T. Fujimoto, and F. Matsuda, Polym. J., 29, 784 (1997).

3. (a) C. M. Starks, C. L. Liotta, and M. Halpern, "Phase-Transfer Catalysis," Chapman \& Hall Inc., New York, N.Y., 1994; (b) T. Nishikubo and T. Iizawa, J. Synth. Org. Chem. Jpn., 51, 157 (1993)

4. (a) T. Shimokawa and T. Nishikubo, Kobunshi Ronbunshu, 44, 641 (1987); (b) T. Shimokawa and T. Nishikubo, Kobunshi Ronbunshu, 49, 577 (1992).

5. (a) T. Nishikubo, T. Iizawa, A. Takahashi, and T. Shimokawa J. Polym. Sci., Part A, Polym. Chem. Ed., 28, 105 (1990); (b) T. Iizawa, K. Nishiyama, and T. Nishikubo, J. Photopolym. Sci. Technol., 3, 125 (1990); (c) T. Iizawa, K. Nishiyama, and T. Nishikubo, J. Polym. Sci., Part A, Polym. Chem. Ed., 31, 443 (1993); (d) T. Iizawa and Y. Sato, Polym. J., 24, 991 (1992); (e) T. Iizawa and E. Seno, Polym. J., 24, 1169 (1992); (f) T. Iizawa and T. Ogasa, J. Polym. Sci., Part A, Polym. Chem. Ed., 34, 63 (1996).

6. (a) S. Yagi and D. Kunii, Kogyo Kagaku Zashi, 53, 131 (1953); (b) O. Levenspiel, "Chemical Reaction Engineering," John Wiley \& Sons, Inc., New York, N.Y., 1972. 